\title{
Heavy quarkonia in a potential model: binding energy, decay width, and survival probability
}

\author{
P. K. Srivastava ${ }^{1, a}$, O. S. K. Chaturvedi ${ }^{2}$, Lata Thakur ${ }^{3, b}$ \\ ${ }^{1}$ Department of Physics, Indian Institute of Technology Ropar, Rupnagar 140001, India \\ ${ }^{2}$ Department of Physics, Institute of Science, Banaras Hindu University, Varanasi 221005, India \\ 3 Theory Division, Physical Research Laboratory, Navrangpura, Ahmedabad 380 009, India
}

Received: 28 November 2017 / Accepted: 19 May 2018 / Published online: 1 June 2018

(C) The Author(s) 2018

\begin{abstract}
Recently a lot of progress has been made in deriving the heavy quark potential within a QCD medium. In this article we have considered heavy quarkonium in a hot quark gluon plasma phase. The heavy-quark potential has been modeled properly for short as well as long distances. The potential at long distances is modeled as a QCD string which is screened at the same scale as the Coulomb field. We have numerically solved the $1+1$-dimensional Schrodinger equation for this potential and obtained the eigen wavefunction and binding energy for the $1 S$ and $2 S$ states of charmonium and bottomonium. Further, we have calculated the decay width and dissociation temperature of quarkonium states in the QCD plasma. Finally, we have used our recently proposed unified model with these new values of decay widths to calculate the survival probability of the various quarkonium states with respect to centrality at relativistic heavy ion collider and large hadron collider energies. This study provides a unified, consistent and comprehensive description of spectroscopic properties of various quarkonium states at finite temperatures along with their nuclear modification factor at different collision energies.
\end{abstract}

\section{Introduction}

Heavy quarkonium production and suppression was one of the earliest proposed tool to study the properties of the medium created in heavy ion collisions. In mid 1980s, Matsui and Satz [1] has proposed theoretically that quarkonium suppression is the signal of the possible creation of quark gluon plasma (QGP) in collision experiments. From there onward, the physical picture of quarkonium dissociation in a thermal medium has undergone various theoretical and experimental

\footnotetext{
a e-mail: prasu111@gmail.com

be-mail: latathakur@prl.res.in
}

refinements [2]. Recent experimental observations suggest that the charmonium suppression in QCD plasma is not the result of a single mechanism, but is a complex interplay of various physical processes. Heavy quarkonia $(Q \bar{Q})$ has a special edge over many other proposed tools since the heavy mass scale $\left(m_{J / \psi}=3.1 \mathrm{GeV}\right.$ for $J / \psi$ and $m_{\Upsilon}=9.2 \mathrm{GeV}$ for $\Upsilon$ ) makes this system possible for analytical treatment theoretically. On the other side, decay of heavy quarkonia via dileptonic channel lead to relatively clean signal which can be precisely measured experimentally.

We can get the physical insight of the medium dependence by analyzing the behavior of spectral function of heavy quarkonium. The two useful approaches to study the production and suppression via spectral function of heavy quarkonium are potential method and lattice approach [3-10]. As we all know that lattice QCD method is first principle tool to study the properties and behavior of heavy quarkonium thus none of the potential method can be alternative to this approach. However, the lattice observations are suffering from discretization effects and statistical errors. In this scenario, potential models can be utilized to serve the purpose. As we now know that the problem of heavy quark bound state at zero temperature involves different energy scales, i.e., hard scale, which is the mass $m_{Q}$ of heavy quark, soft scale which is inverse size $m_{Q} v \sim 1 / r$ of bound state and ultrasoft scale, which is the binding energy $m v^{2} \sim \alpha_{s} / r$. After integrating out the hard scale modes, one obtains an effective field theory non-relativistic QCD (NRQCD) [11,12]. Subsequently, integrating out the modes related with the inverse size scale, potential NRQCD (pNRQCD) appears [13,14]. In this pNRQCD, the heavy quark-antiquark pair in singlet and octet state are included via dynamical singlet and octet fields (or potentials).

The generalization of this approach at finite temperature involves three different thermal scales : $T, g T$ and $g^{2} T$. In the static limit and if the binding energy is larger than the 
temperature $T$, the derivation of $\mathrm{pNRQCD}$ proceed in the same way as in zero temperature theory and heavy quark potential is not affected by the medium. However, the bound state properties can be affected through interaction of bound states with the ultrasoft gluons of the medium. The main effect of this interaction is the reduction of binding energy of the heavy quark bound state and emergence of a finite thermal width. In second case when one of the thermal scales is higher than binding energy, the singlet and octet potential become temperature dependent and will acquire an imaginary part [15]. It is important to state here that the real part of the potential leads to colour screening while imaginary part of the potential introduces the Landau damping to the heavy quark bound states [16-19].

Another observation from numerical lattice calculations show the crossover type of deconfinement transition from hadron gas to QGP [20]. Thus, we can expect some nonperturbative effects such as non-vanishing string tension in heavy quark-antiquark potential above the critical temperature $T_{C}$ as well. So it is reasonable to assume the string term above $T_{C}$ [21-23]. Further this potential should also incorporate the effect of Landau damping induced thermal width by calculating the imaginary part of the potential. In the recent years, the real $[24,25]$ and imaginary parts $[17,26]$ of the heavy quark potential have been calculated by modifying both the perturbative and non-perturbative terms of the Cornell potential in the static as well as in a moving medium. The complex static interquark potential at finite temperature has also been derived in Ref. [27] by considering both the coulombic and linear string terms. One can calculate the dissociation coefficient at a given temperature by solving the Schrödinger equation using the modified heavy quark complex potential $[17,24,25,28,29]$. Recently we have constructed a unified model for charmonium suppression in Ref. [30]. Here we want to incorporate this modified heavy quark potential from Refs. [17,24] in unified model to calculate the survival probability of heavy quarkonium states. The survival probability of heavy quarkonium states has been studied recently in Refs. [31,32]. Specially we will focus here on the double ratio of two states of charmonium since most of the suppression models are failed to reproduce the suppression pattern of this double ratio.

In this article we have modified our unified model to properly include perturbative as well as nonperturbative effects on quarkonium suppression. We have constructed this model based on the kinetic approach whose original ingredients was given by Thews et al. [33-35]. In this approach, there are two terms written on the basis of Boltzmann kinetic equation as shown in Sect. 2.4. First term, which we call as dissociation term, includes the dissociation process like gluo-dissociation and collisional damping. The second term (formation term) provides the (re)generation of $J / \psi$ due to the recombination of charm-anticharm quark. These two terms compete over the entire temporal evolution of the QGP and we get the multiplicity of finally survived quarkonia at freezeout temperature. To define the dynamics of the system created in the heavy ion collisions, we have used the $1+1$ dimensional viscous hydrodynamics. Here we have included only the shear viscosity and neglected the bulk viscosity. We have also suitably incorporated the overall feed-down correction from the higher states to the low-lying states. Rest of the paper is organised as follows: in Sect. 2, model formulation, we have provided four subsections which discuss briefly about modified heavy quark potential at finite temperature, binding energy, decay width and calculation of survival probability, respectively. Further in Sect. 3, we have presented our results along with their discussions. In the end of this section, we have also summarized our present work.

\section{Model formalism}

\subsection{Heavy quark complex potential}

In this section we discuss about the heavy quark-antiquark potential which have both the coulombic and string-like parts. Authors in Ref. [16] have derived the static potential between heavy quark-antiquark pair at finite temperature by defining a suitable gauge-invariant Green's function and computing it to first order in hard thermal loop (HTL) resummed perturbation theory. In medium both the coulombic and stringlike part of potential receive modification. Further complications arise from the fact an imaginary part of the potential arises due to the presence of scattering of light medium degrees of freedom with the color string spanning in between the heavy quarks and antiquarks. It has been pointed out that the physics of the finite width originates from the Landau damping of low-frequency gauge fields. Further it has been studied non-perturbatively by making use of the classical approximation. In the view of above observations, a meaningful description of the relevant physics of quarkonium must therefore consist both the effects of screening of the real part of potential and imaginary part of the potential. There are several efforts to derive and/or phenomenologically construct HQ potential which can be used as an input in the quarkonium suppression models [16,27,36-40]. The standard Polyakov loop correlator is fail to reproduce the expected Debye-screening potential at asymptotically large distances. Many modified descriptions of Polyakov loop correlator are affected by gauge ambiguities. There are studies based on generalized Gauss law and further its combination with the characterization of in-medium effects through the perturbative HTL permittivity. The use of Gauss law, non-local concept leads to a self-consistent descriptions of both screening and damping effects. Recently, direct lattice determination of the quarkonium spectral function have 
been attempted [10]. However, these calculations are again plagued by the model assumptions as there are very finite number of points in time direction and data is of statistical nature in these lattice studies. On the other side, similar observables have been calculated for strongly-coupled $N=4$ super Yang-Mills theory through AdS/CFT correspondence [41-45]. In these derivations, static potentials in real time can be calculated by computing the standard Wilson loop in Euclidean spacetime, and then carry out the analytic continuation. In other words, the expectation value of a particular timelike Wilson loop defines the potential between a static quark and antiquark at finite temperature. The medium modified heavy quark potential can be obtained by correcting both the coulombic (short-distance) and string (long-distance) terms, not its Coulomb term alone, with a dielectric function encoding the effects of the deconfined medium as discussed in Refs. [17,24,25]. In the literature only a screened Coulomb potential was assumed above critical temperature $\left(T_{c}\right)$ and the non-perturbative (string) term was usually overlooked (assumed zero), was certainly worth investigation. Recent lattice results indicate the phase transition in full QCD appears to be a crossover rather than a phase transition with the related singularities in thermodynamic observables as discussed in introduction section. The effects of string tension between the $Q \bar{Q}$ pairs should not be ignored beyond $T_{c}$. Therefore, it is important to incorporate the string term while setting up the criterion for the dissociation. In our approach, we make the assumption that medium potential can be derived from the vacuum potential by multiplying it with a field-theoretically determined complex permittivity in momentum space. It is possible to reproduce the real and imaginary part of the corresponding in-medium potential in by using the hard thermal loop permittivity. The real part of the medium modified heavy quark potential can be written as [24,25]

$\operatorname{ReV}(r, T)=-\alpha m_{D}\left(\frac{e^{-\hat{r}}}{\hat{r}}+1\right)+\frac{2 \sigma}{m_{D}}\left(\frac{e^{-\hat{r}}-1}{\hat{r}}+1\right)$,

where $\hat{r}=r m_{D}$ and $\alpha=4 / 3 \alpha_{s}$ with $\alpha_{s}$ as the one loop running coupling constant given as

$\alpha_{S}(T)=\frac{g_{s}^{2}(T)}{4 \pi}=\frac{6 \pi}{\left(33-2 N_{f}\right) \ln \left(\frac{2 \pi T}{\Lambda_{\overline{\mathrm{MS}}}}\right)}$.

Here we take $\Lambda_{\overline{\mathrm{MS}}}=0.1 \mathrm{GeV}$ and the string tension, $\sigma=0.184 \mathrm{GeV}^{2} \cdot m_{D}$ is the Debye screening mass which is defined as

$m_{D}^{2}=\frac{g^{2} T^{2}}{6}\left(N_{f}+2 N_{c}\right)$,

with $N_{f}$ and $N_{c}$ as the number of flavours and colours, respectively.
In the small $r$ limit, the real part of potential reduces to the Cornell potential.

$\operatorname{ReV}(r) \approx-\frac{\alpha}{r}+\sigma r$.

On the other hand, in the large distance limit (where the screening occurs), potential is reduced to a longrange Coulomb potential with a dynamically screened-color charge. However, if we compare our $Q \bar{Q}$ potential (Eq. 1) with the classical concept of Debye-Hückel theory by Digal et al. [46], we found that in the asymptotic limit $(r \rightarrow \infty)$, Eq. (1) reduces to

$\operatorname{ReV}(r \rightarrow \infty, T)=F(\infty, T)=\frac{2 \sigma}{m_{D}}-\alpha m_{D}$,

whereas in Ref. [46] free energy reduces to

$F^{\text {Digal }}(\infty, T)=\frac{\Gamma(1 / 4)}{2^{3 / 2} \Gamma(3 / 4)} \frac{\sigma}{m_{D}}-\alpha m_{D}$,

here the difference can be seen only in the string term only and may be due to the treatment of the problem classically or quantum mechanically. Also in the framework of DebyeHückel theory, Digal et al. employed different screening functions, $f_{c}$ and $f_{s}$ for the Coulomb and string terms, respectively, to obtain the free energy. Here we have used the same screening scale, $m_{D}$ for both the coulombic and linear terms.

The imaginary part of the medium modified heavy quark potential can be calculated in the similar way as in Ref. [17] and is given by

$\operatorname{Im} V(r, T)=-\alpha T \phi\left(m_{D} r\right)-\frac{2 \sigma T}{m_{D}^{2}} \psi\left(m_{D} r\right)$,

where the functions $\phi(\hat{r})$ and $\psi(\hat{r})$ are defined as

$\phi(\hat{r})=2 \int_{0}^{\infty} \frac{z d z}{\left(z^{2}+1\right)^{2}}\left(1-\frac{\sin z \hat{r}}{z \hat{r}}\right)$

and

$\psi(\hat{r})=2 \int_{0}^{\infty} \frac{d z}{z\left(z^{2}+1\right)^{2}}\left(1-\frac{\sin z \hat{r}}{z \hat{r}}\right)$,

In the small $r$ limit, we can expand the potential and at leading logarithmic order in $\hat{r}$ we get

$$
\begin{aligned}
\operatorname{Im} V(\hat{r}, T) \approx- & \alpha T \frac{\hat{r}^{2}}{3} \log \left(\frac{1}{\hat{r}}\right) \\
& -\frac{2 \sigma T}{m_{D}^{2}}\left(\frac{\hat{r}^{2}}{6}-\frac{\hat{r}^{4}}{60}\right) \log \left(\frac{1}{\hat{r}}\right) .
\end{aligned}
$$

\subsection{Binding energy}

Binding energy can be calculated by knowing the energy eigen value of different quarkonia state. The binding energy here is a function of temperature instead of a constant factor. To know the energy eigen value and energy eigen function of different quarkonia states, we have solved the Schrodinger 
equation with the real part of heavy quark potential as described above and a angular momentum dependent part. The motivation behind using only the real part of the potential in solving Schrodinger equation is the large magnitude of real part over imaginary part (one can easily verify it from Figs. 1 and 2). We have used the method of Ganesh and Mishra [29] to numerically solve the one dimensional Schrodinger equation on a logarithmic equally spaced one dimensional lattice. We have obtained the energy eigen values and eigen functions of different quarkonium states. Then we calculate the energy eigen value at infinity $\left(U_{\infty}\right)$. The binding energy of a given quarkonia state with principal quantum number $n$ and orbital quantum number $l$ is calculated by using the following relation [47]

$$
\text { B.E. }(n, l)=g_{n l}-U_{\infty} .
$$

\subsection{Decay width $(\Gamma)$}

We have calculated the decay width $(\Gamma)$ of $1 S$ and $2 S$ of quarkonia state numerically. As we know that the thermal width can be calculated from the imaginary part of the potential by using the following expression $[17,26,42]$

$\Gamma=4 \pi \int g_{n l}^{*}[\operatorname{Im} V] g_{n l} r^{2} d r$

It is important to mention here that analytically one can calculate the decay width by folding the imaginary part of the potential with $1 \mathrm{~S}$ and $2 \mathrm{~S}$ hydrogen atom wavefunction $\left(g_{1 S}=\frac{1}{\sqrt{\pi a_{0}^{3}}} \exp \left(-\frac{r}{a_{0}}\right)\right.$, and $g_{2 S}=\frac{1}{\sqrt{32 \pi a_{0}^{3}}}\left(2-\frac{r}{a_{0}}\right) \exp$ $\left.\left(-\frac{r}{2 a_{0}}\right)\right)$ which are assumed to represent most of the properties of heavy quarkonia states. However as we will show in our results that the various quarkonia wavefunctions actually depends on temperature very strongly and thus assuming a temperature independent coulombic wave function to calculate the decay width is not realistic. Thus we have used the wavefunctions as obtained by us, solving Schrodinger equation at different temperatures.

For the sake of comparison, we are providing here the expression of decay widths obtained by folding the imaginary part of the potential with the coulombic wavefunctions of different quarkonia state as follows:

$$
\begin{aligned}
\Gamma_{1 S}= & \frac{4 T}{\alpha m_{Q}^{2}} m_{D}^{2} \log \left(\frac{\alpha m_{Q}}{m_{D}}\right) \\
& +\frac{4 \sigma T}{\alpha^{2} m_{Q}^{2}}\left[1-\frac{3 m_{D}^{2}}{\alpha^{2} m_{Q}^{2}}\right] \log \left(\frac{\alpha m_{Q}}{m_{D}}\right),
\end{aligned}
$$

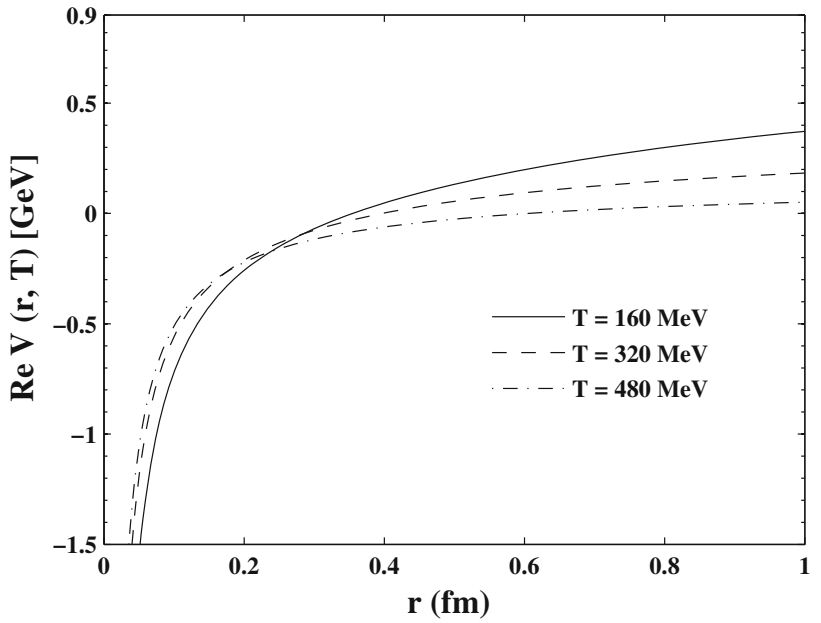

Fig. 1 Variation of the real part of potential with the separation distance $r$ between the $Q \bar{Q}$ pair at three different values temperatures, i.e., $T=$ 160, 320 and $480 \mathrm{MeV}$

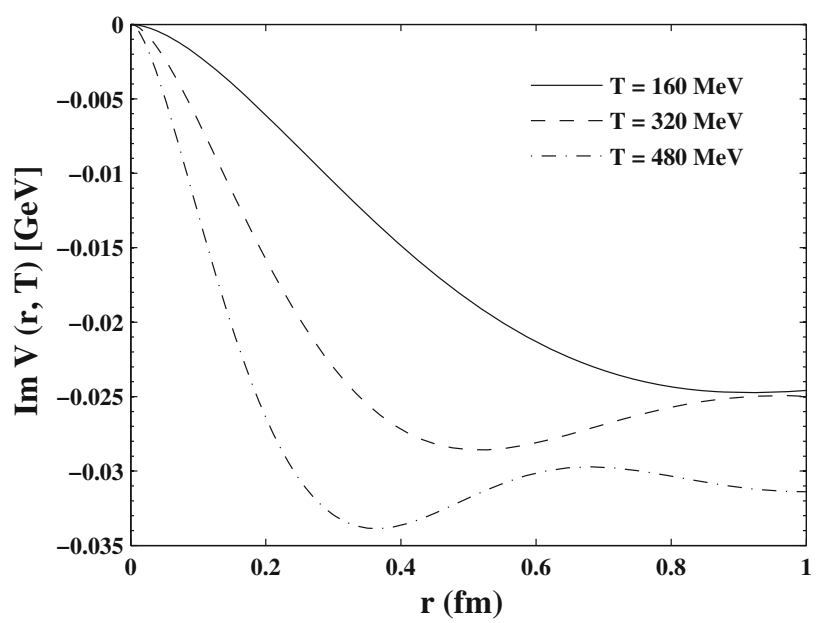

Fig. 2 Variation of the imaginary part of potential with the separation distance $r$ at three different values of temperature

and

$$
\begin{aligned}
\Gamma_{2 S}= & \frac{56 T}{\alpha m_{Q}^{2}} m_{D}^{2} \log \left(\frac{\alpha m_{Q}}{m_{D}}\right) \\
& +\frac{8 \sigma T}{\alpha^{2} m_{Q}^{2}}\left[7-\frac{192 m_{D}^{2}}{\alpha^{2} m_{Q}^{2}}\right] \log \left(\frac{\alpha m_{Q}}{2 m_{D}}\right),
\end{aligned}
$$

The dissociation temperature for the quarkonium states can be determined by using the conservative quantitative condition $\Gamma_{n, l}\left(T_{C}\right) \approx 2 \times B \cdot E_{\cdot n, l}\left(T_{C}\right)$ [7], where $T_{C}$ is the dissociation temperature of that particular quarkonia state having principal quantum number $n$ and azimuthal quantum number $l$. 


\subsection{Survival probability including regeneration}

The survival probability or the nuclear modification factor of various quarkonia states with respect to centrality, rapidity and transverse momentum is key signatures to quantify the properties of medium created in heavy ion collision experiments. As we have the decay width of various quarkonia states from our calculation, we can calculate their survival probability by using our recently proposed unified model [30]. Here we show the variation of survival probability with respect to participant number $\left(N_{\text {part }}\right)$ which actually measures the centrality of collision. To understand the evolution dynamics of the system created in heavy ion collisions, we have used $1+1$ dimensional viscous hydrodynamics in which we have included the shear viscous effect. We have done our calculation for mid-rapidity region only where Bjorken scaling are applicable. We have derived the cooling law for temperature $[48,49]$ which depends only on proper time $\tau$ and then extend this cooling law to make it $\tau$ and $N_{\text {part }}$ dependent using Refs. [29,50]. To calculate the survival probability we have used the following expression:

$S=\frac{N_{H M}^{f}}{N_{H M}^{i}}$

where $N_{H M}^{i}$ and $N_{H M}^{f}$ is the initial and final multiplicity of heavy meson (quarkonia). The final multiplicity of quarkonia can be calculated as [30]:

$N_{H M}^{f}=\epsilon\left(\tau_{f}\right)\left[N_{H M}^{i}+N_{Q \bar{Q}}^{2} \int_{\tau_{0}}^{\tau_{f}} \Gamma_{f}(V(\tau) \epsilon(\tau))^{-1} d \tau\right]$.

Here $\Gamma_{f}$ is the reactivity for the recombination of uncorrelated $Q$ and $\bar{Q}$ quark to form a quarkonia and it can be calculated by using decay width [30]. $N_{Q \bar{Q}}$ is the number density of quark-antiquark pair. $\epsilon\left(\tau_{f}\right)$ is the dissociation factor which can be calculated using the following expression :

$\epsilon\left(\tau_{f}\right)=\exp \left(-\int_{\tau_{0}}^{\tau_{f}} \Gamma d \tau\right)$

where $\tau_{0}$ and $\tau_{f}$ are initial and final proper time which actually spans over the QGP lifetime, i.e., $\tau_{0}=0.5 \mathrm{fm}$ and $\tau_{f}=6.0 \mathrm{fm}$. We have used $\tau$ and $N_{\text {part }}$ dependent cooling law for temperature as follows $[48,50]$ :

$T(\tau)=T_{c}\left(\frac{N_{\text {part }}(\text { bin })}{N_{\text {part }}\left(\text { bin }_{0}\right)}\right)^{1 / 3}\left(\frac{\tau_{Q G P}}{\tau}\right)^{1 / 3}$,

where $N_{\text {part }}\left(\operatorname{bin}_{0}\right)$ is the number of participant corresponding to the most central bin as used in our calculation and $N_{\text {part }}($ bin) is the number of participant corresponding to the bin at which we want to calculate the temperature. $\tau_{Q G P}$ is the lifetime of QGP.
The cooling law for volume is derived using the condition of isentropic evolution of the medium and can be expressed as follows [30]:

$V(\tau, b)=V\left(\tau_{0}, b\right)\left(\frac{\tau_{0}}{\tau}\right)^{\left(\frac{1}{R}-1\right)}$

where $V\left(\tau_{0}, b\right)=\pi\left(r_{t}-b / 2\right)^{2} \tau_{0}$ is volume at the initial time $\tau_{0}$ and an impact of $b \mathrm{fm}$.

$N_{c \bar{c}}$ and $N_{b \bar{b}}$ are calculated in our model using the help of Glauber model. The extrapolation to the nucleus-nucleus collisions is done via standard overlap integral scaling as follows:

$N_{c \bar{c}}(b)=\sigma_{c \bar{c}}^{N N} T_{A A}$

where $\sigma_{c \bar{c}}^{N N}$ is the cross section for $c \bar{c}$ pair production in $\mathrm{p}+\mathrm{p}$ collision. The $\sigma_{c \bar{c}}^{N N}$ has been calculated using pQCD approach for GRV HO hadronic structure function [33-35], we have obtained $\sigma_{c \bar{c}}^{N N}=3.546 \mathrm{mb}$ and $\sigma_{b \bar{b}}^{N N}=0.1105 \mathrm{mb}$ for large hadron collider (LHC) at $\sqrt{s}=2.76 \mathrm{TeV}$. Further we have obtained $\sigma_{c \bar{c}}^{N N}=0.346 \mathrm{mb} \sigma_{b \bar{b}}^{N N}=0.01035 \mathrm{mb}$ for relativistic heavy ion collider (RHIC) at $\sqrt{s}=200 \mathrm{GeV}$. Here, $T_{A A}(b)$ is nuclear overlap function, its impact parameter $(b)$ dependent values have been taken from Ref. [51]. Here it is important to mention that we have not incorporated any type of cold nuclear matter (CNM) effect in the present calculations.

\section{Results and discussions}

The main ingredient of this paper is the heavy quark potential in QCD plasma [17,24]. We first show few characteristics of this potential. In Fig. 1, we demonstrate the variation of the real part of the heavy-quark potential with respect to the separation distance $(r)$ between the $Q \bar{Q}$ pair. We have plotted the real part of potential at three different values of temperature, i.e., $T=160,320$ and $480 \mathrm{MeV}$ by solid, dashed and dash-dotted curve, respectively. The real potential starts from negative value and increases very sharply to zero as we increase the distance from zero to 0.5 fermi. Further the real part of potential increases from zero to $0.4 \mathrm{GeV}$ as we increase the distance at $T=160 \mathrm{MeV}$. We choose this specific value of temperature since we take $160 \mathrm{MeV}$ as the critical crossover temperature $\left(T_{C}\right)$ in our calculation. We also show the variation in the saturation value of real potential with increase in temperature. As we increase the temperature the potential saturates at lower values, i.e., $0.4 \mathrm{GeV}$ at $T=160 \mathrm{MeV}$ to $0.05 \mathrm{GeV}$ at $T=480 \mathrm{MeV}$. In Fig. 2, we have plotted the imaginary part of heavy-quark potential with respect to $r$. The imaginary potential starts from zero value at $r=0 \mathrm{fm}$ and then decreases and became negative with increase in $r$. As we increase the temperature the mag- 


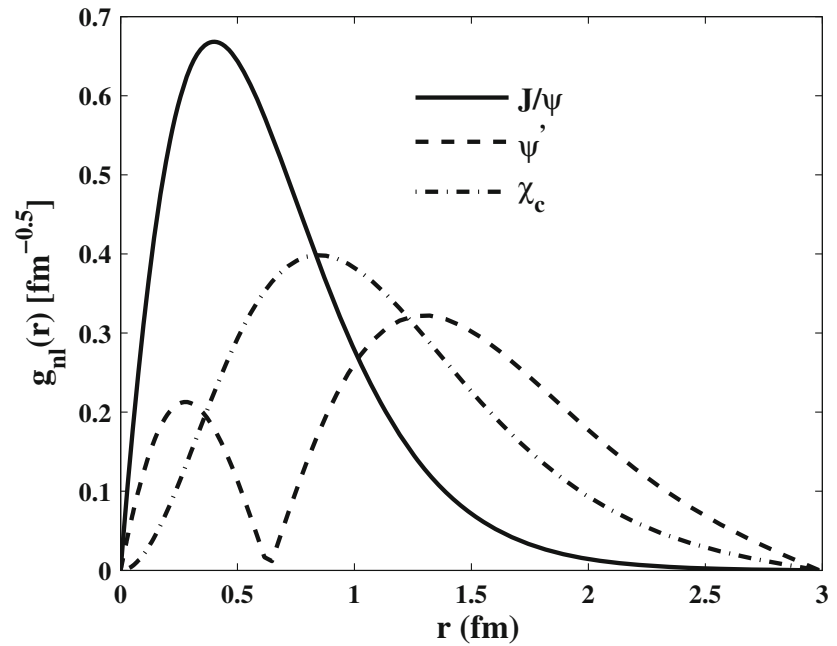

Fig. 3 The variation of radial part of wavefunction of different charmonia states with respect to $r$. Solid curve represents the $1 S(J / \psi)$, dashed curve is for $2 S\left(\psi^{\prime}\right)$ and dash-dotted curve represents the radial wavefunction of $1 P\left(\chi_{c}\right)$ state

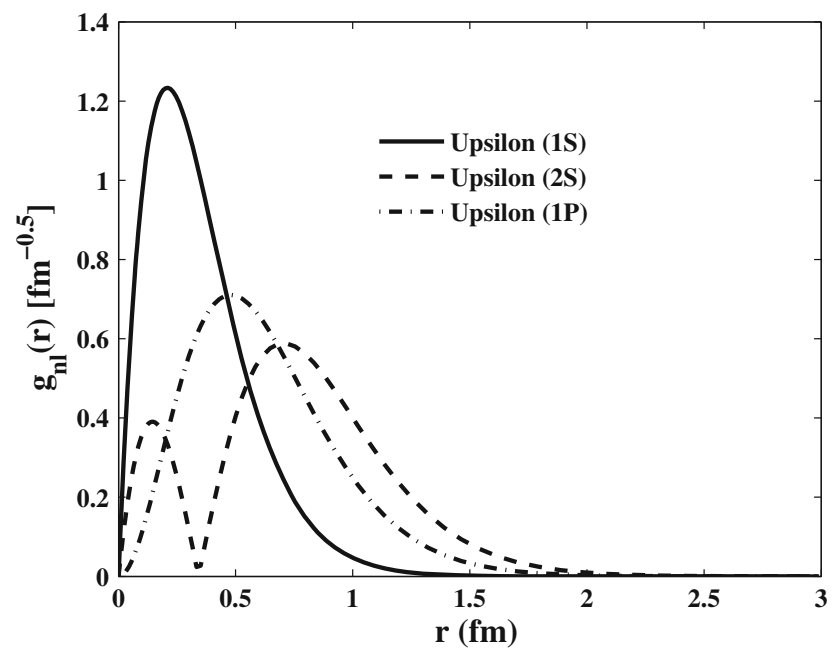

Fig. 4 The variation of radial part of wavefunction of different bottomonia states with respect to $r$. Solid curve represents the $1 S(\Upsilon)$, dashed curve is for $2 S(\Upsilon)$ and dash-dotted curve represents the radial wavefunction of $1 P\left(\chi_{b}\right)$ state

nitude of imaginary potential also increases in the negative direction. At higher temperatures, we observed a fluctuating behaviour for $r>0.4 \mathrm{fm}$. This is due to the sine term in imaginary potential.

Figure 3 represents the variation of radial part of eigen wavefunction for a given state $n$ and $l$, i.e., $g_{n l}(r)$ of $J / \Psi$ and $\Psi^{\prime}$ and $\chi_{C}$ states with respect to the $r$ at the critical temperature, $T_{C}$. Similarly, Fig. 4 demonstrates the radial part of wavefunction of different bottomonia states and their variation with respect to $r$ at $T_{C}$.

Figure 5 presents a comparison between eigen wave function of charmonium $(J / \Psi)$ and bottomonium $(\Upsilon(1 S))$. Here

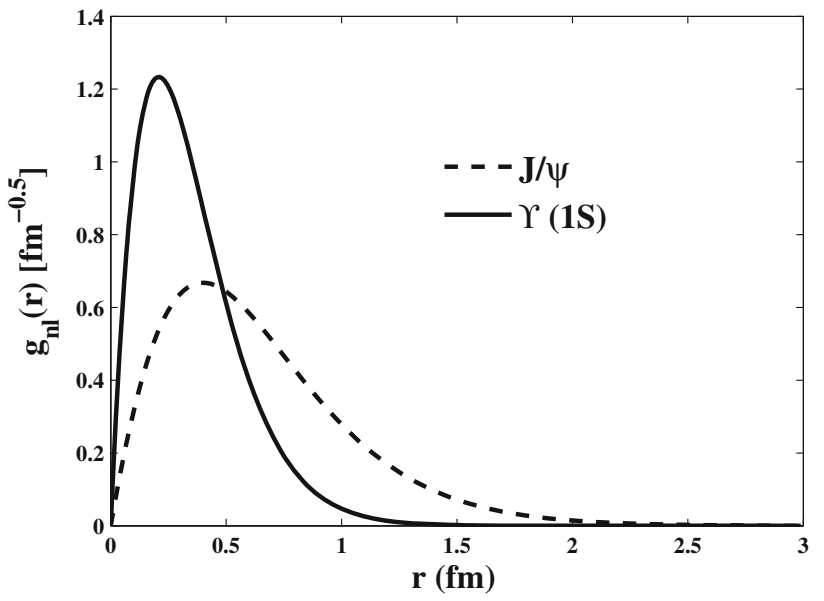

Fig. 5 Comparison of radial part of wavefunction of $\Upsilon(1 S)$ and $J / \psi$ at critical temperature $T_{C}=160 \mathrm{MeV}$

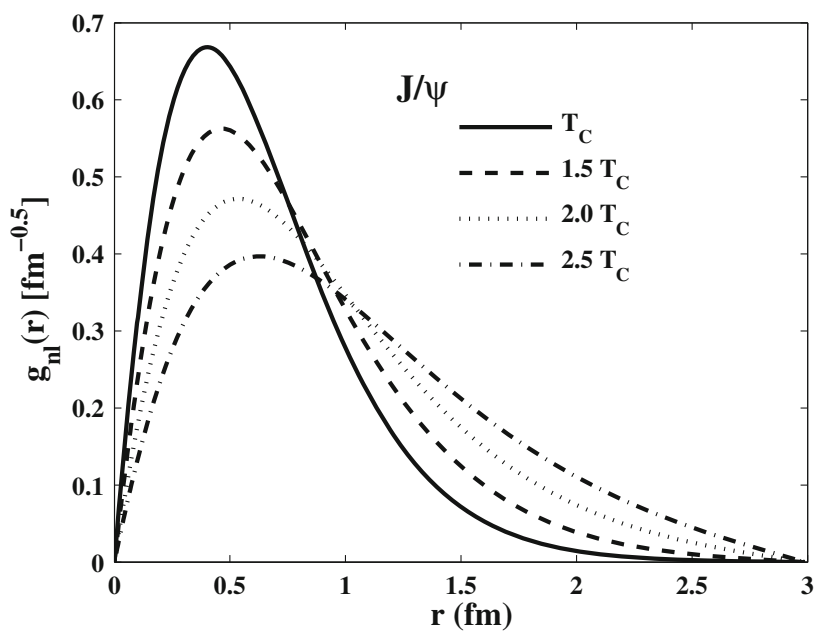

Fig. 6 Variation of radial wavefunction of $J / \psi$ with respect to $r$ at $T=T_{C}, 1.5 T_{C}, 2.0 T_{C}$ and $2.5 T_{C}$

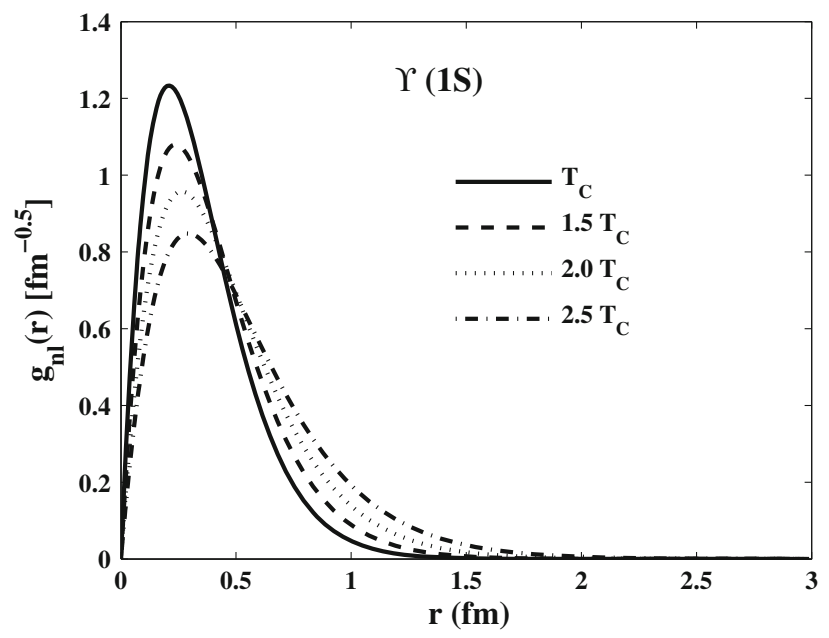

Fig. 7 Variation of radial wavefunction of $\Upsilon(1 S)$ with respect to $r$ at $T=T_{C}, 1.5 T_{C}, 2.0 T_{C}$ and $2.5 T_{C}$ 


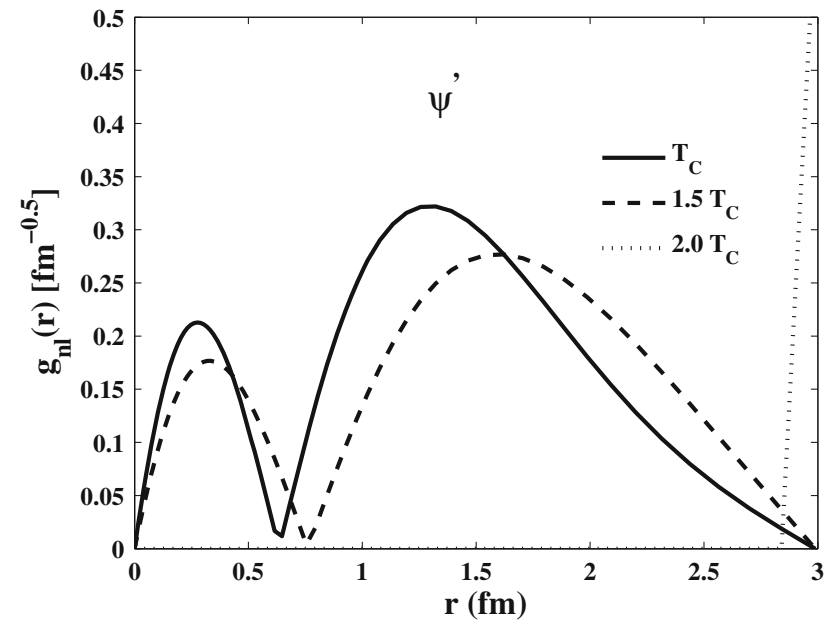

Fig. 8 Variation of radial wavefunction of $\psi^{\prime}$ with respect to $r$ at $T=$ $T_{C}, 1.5 T_{C}, 2.0 T_{C}$ and $2.5 T_{C}$

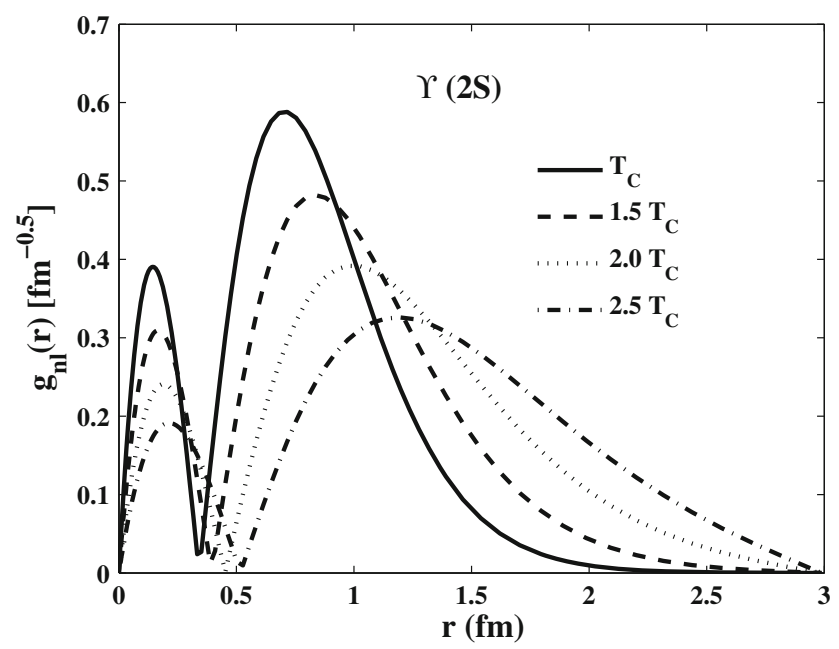

Fig. 9 Variation of radial wavefunction of $\Upsilon(2 S)$ with respect to $r$ at $T=T_{C}, 1.5 T_{C}, 2.0 T_{C}$ and $2.5 T_{C}$

one can clearly see the difference in peak height and peakwidth of $J / \Psi$ and $\Upsilon(1 S)$ and understand the strong binding of $b-\bar{b}$ quark in $\Upsilon(1 S)$ in comparison to the binding of $c-\bar{c}$ quark in $J / \Psi$.

Figure 6 shows the change in the radial part of wavefunction of charmonium as we increase the temperature from $T_{C}$ to $2.5 T_{C}$ in the step of $0.5 T_{C}$. From here it is clear that as we increase the temperature the peak-height of eigen function decreases and the peak-width increases which causes the binding between the heavy quark and anti-quark in the bound state of charmonium to decrease. Similarly we have shown the change in eigen function of $\Upsilon(1 S)$ with respect to temperature in Fig. 7.

In Figs. 8 and 9, we demonstrate the change in shape of eigen functions of $\Psi^{\prime}$ and $\Upsilon(2 S)$ state with respect to temperature going from $T_{C}$ to $2.5 T_{C}$, respectively. Figure 10

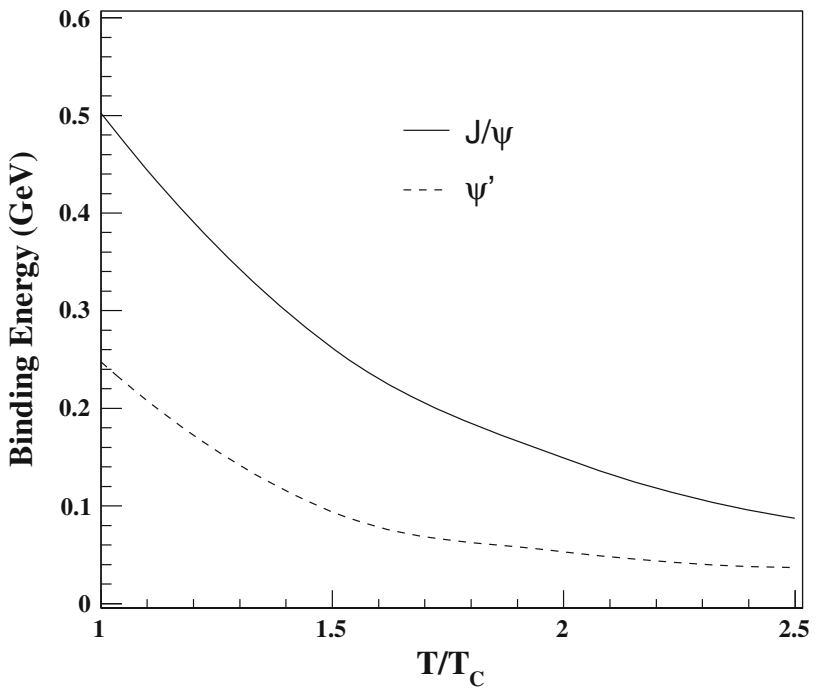

Fig. 10 Variation of binding energy (B.E.) with respect to temperature in units of $T_{C}$. Solid and dashed curves represent the binding energy of $J / \psi$ and $\psi^{\prime}$, respectively

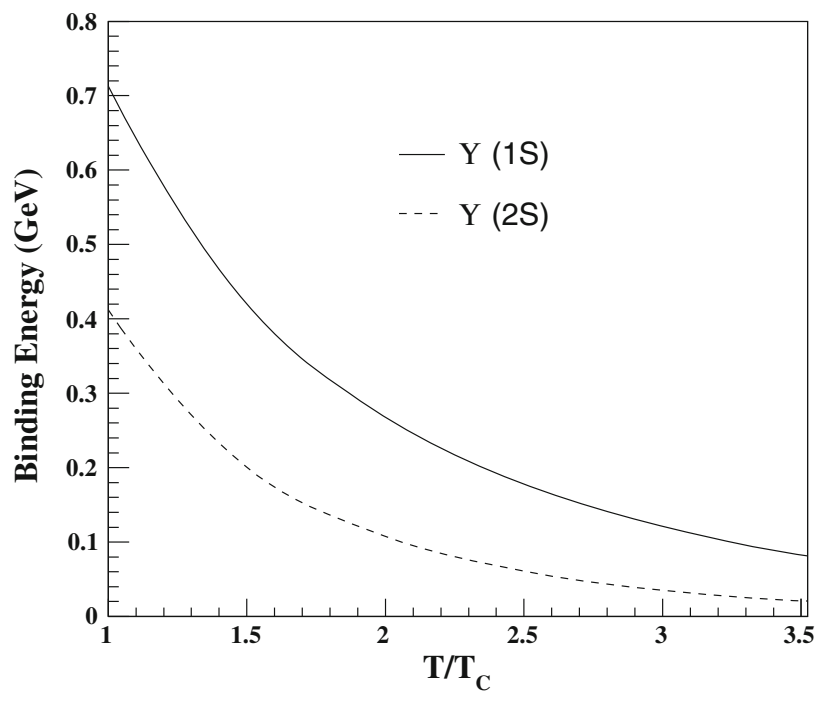

Fig. 11 Variation of binding energy (B.E.) with respect to temperature in units of $T_{C}$. Solid and dashed curves represent the binding energy of $\Upsilon(1 S)$ and $\Upsilon(2 S)$, respectively

represents the variation of binding energy of $J / \Psi$ and $\Psi^{\prime}$ with respect to temperature. Here we present the temperature in the unit of $T_{C}$. It is clear from the plot that initially when the temperature is near $T_{C}$, the binding energy of $J / \Psi$ is large and thus charmonium can still survive after critical temperature. As we start to increase the temperature from $T_{C}$ to higher values, the binding energy of charmonium starts decreasing and acquires a low value which is near to zero. However, our calculation shows that even at $2.5 T_{C}$, there is a finite value of binding energy for $J / \Psi$. For $\Psi^{\prime}$, the binding energy starts from a lower value in comparison to $J / \Psi$ which 


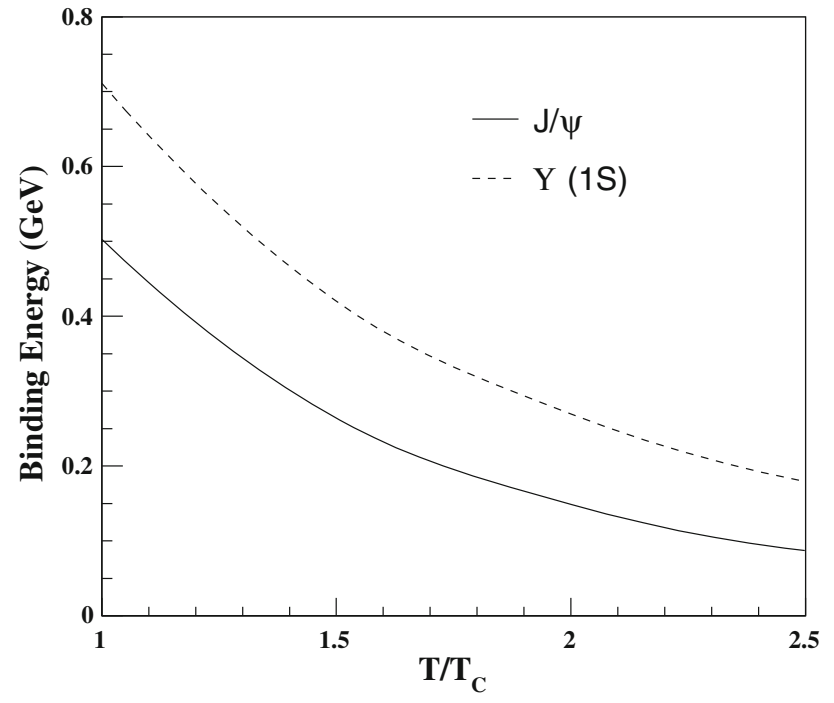

Fig. 12 Comparison of binding energy of $\Upsilon(1 S)$ and $J / \psi$

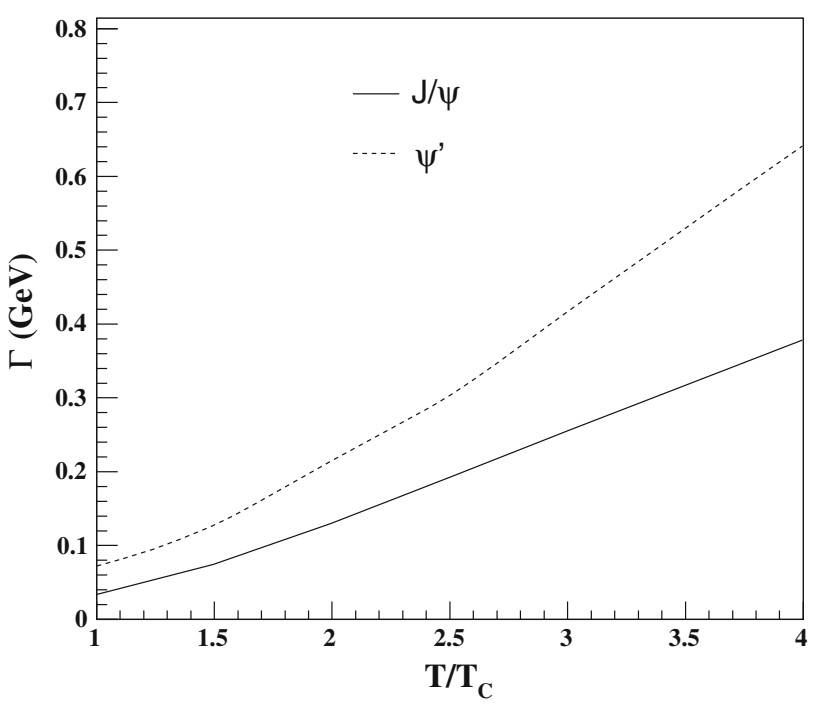

Fig. 13 Variation of decay width $(\Gamma)$ with respect to $T / T_{C}$. Solid and dash-dotted curves represent the decay width of $J / \psi$ and $\psi^{\prime}$, respectively

is quite obvious and it decreases with increase in temperature and acquire almost zero value at $2.0 T_{C}$.

Bottomonia wavefunction is more coulombic at $T_{C}$ in comparison to charmonia states due to the large mass of $\Upsilon(1 S)$ and $\Upsilon(2 S)$ in comparison to $J / \Psi$ and $\Psi^{\prime}$. Therefore the binding energy of various bottomonia states start from a higher value in comparison to corresponding charmonia states. Figure 11 represents the variation of binding energy of $\Upsilon(1 S)$ and $\Upsilon(2 S)$ with respect to $T / T_{C}$. Further, Fig. 12 shows the comparison of binding energy of charmonia $1 S$ state with bottomonia $1 S$ state.

We now present the decay width coming only due to the imaginary part of the heavy quark potential. In Fig. 13, we

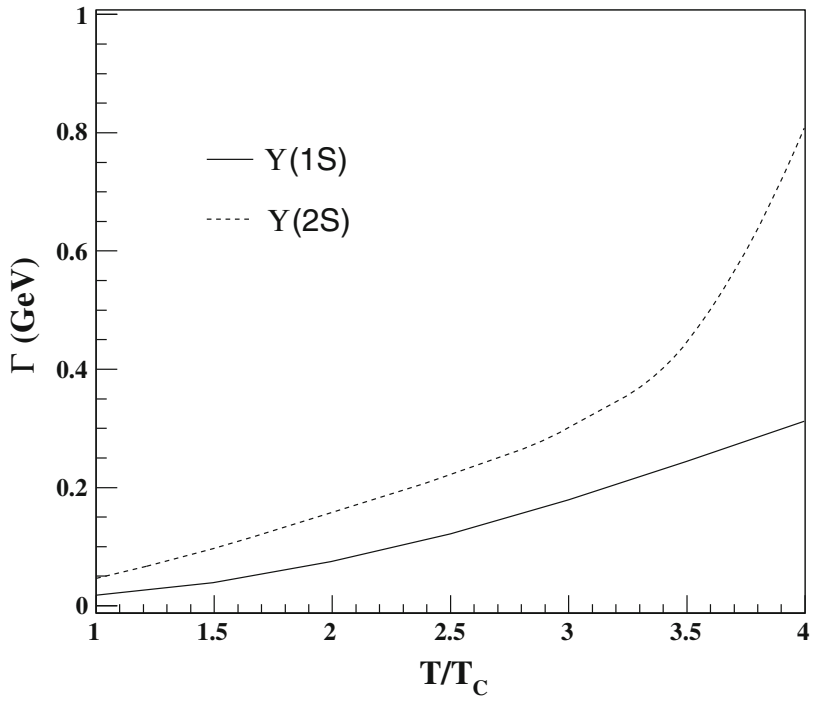

Fig. 14 Variation of decay width $(\Gamma)$ with respect to $T / T_{C}$. Solid and dashed curves represent the decay width of $\Upsilon(1 S)$ and $\Upsilon(2 S)$, respectively

demonstrate the variation of decay width of $1 \mathrm{~S}$ and $2 \mathrm{~S}$ charmonia states with respect to $T / T_{C}$. As we have earlier shown in Fig. 10 that the binding energy of $J / \Psi$ is large at $T_{C}$ and thus the decay width should be small at $T_{C}$. As the binding energy of $\Psi^{\prime}$ is less in comparison to $J / \Psi$ over the entire temperature range therefore $\Psi^{\prime}$ has a larger decay width than $J / \Psi$ as shown in this figure at each temperature. Further, the decay width increases with the increase in temperature. Furthermore, the difference between the decay width of $J / \Psi$ and $\Psi^{\prime}$ increases with the temperature.

In Fig. 14, we have plotted the variation of decay width of $\Upsilon(1 S)$ and $\Upsilon(2 S)$ with respect to $T / T_{C}$. The trend is quite similar with the charmonia states but the decay width of $\Upsilon(2 S)$ increases rapidly after $T=3.5 T_{C}$. Figure 15 presents a comparison between the decay width of $J / \Psi$ and $\Upsilon(1 S)$. This figure clearly shows that decay width of $J / \Psi$ and $\Upsilon(1 S)$ starts from almost similar value at $T_{C}$. However, the width of $J / \Psi$ increases more rapidly in comparison to $\Upsilon(1 S)$. This means that the $J / \Psi$ dissociates at lower temperatures in comparison to bottomonium which is a tightly bound state and thus survive to higher temperatures.

After that we have obtained the dissociation temperature for the different quarkonium states. Different dissociation criteria have been discussed in the literature. The first criteria is that a quarkonium state should dissociate at the temperature $T$ where B.E. $=T$. Here B.E. is the binding energy of that particular quarkonia state. This criteria can provide an upper bound on the dissociation temperature. Here we use a more strict dissociation criteria which suggest that any quarkonium state should dissociate at that temperature where the decay width of the quarkonium state becomes equal to two times of its binding energy, i.e., $\Gamma=2$ B.E. [7,17]. 


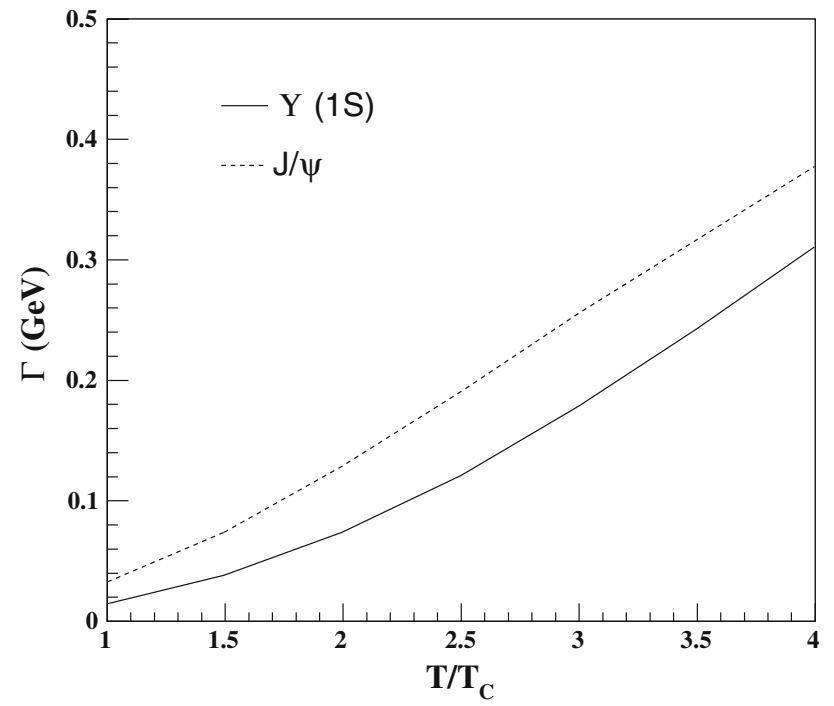

Fig. 15 Comparison of decay width of $J / \psi$ and $\Upsilon(1 S)$

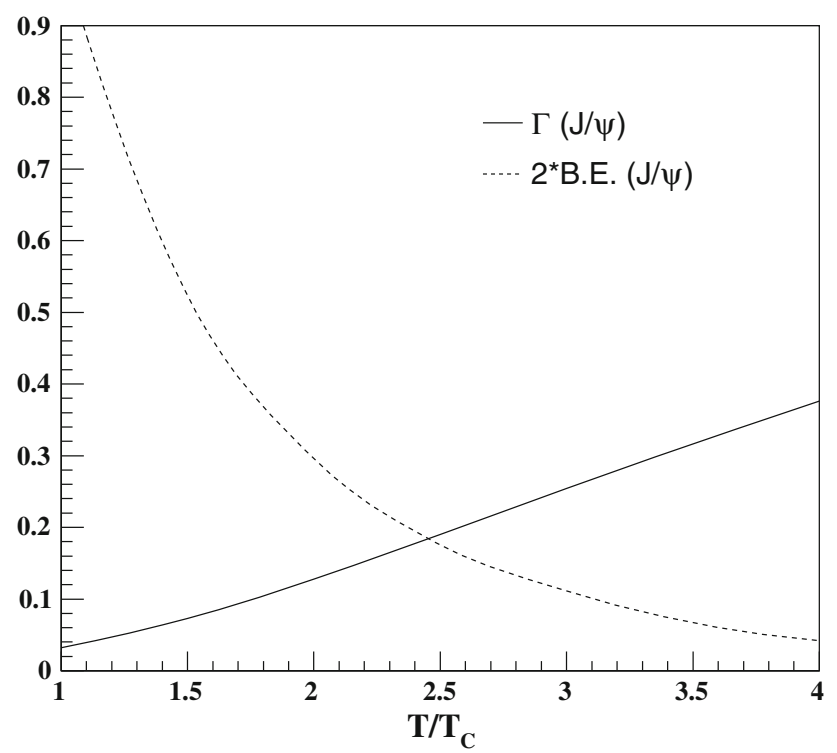

Fig. 16 Variation of two times of binding energy of $J / \psi$ and decay width with respect to $T / T_{C}$

In Fig. 16 we have plotted the decay width and two times of binding energy of $J / \Psi$ with respect to $T / T_{C}$. The two curves intersect each other at $2.4 T_{C}$. Thus the dissociation temperature of $J / \Psi$ comes out as $2.4 T_{C}$ in our calculation. We have plotted the width and the two times of binding energy of $\Psi^{\prime}$ with respect to $T / T_{C}$ in Fig. 17. The dissociation temperature of $\Psi^{\prime}$ as obtained from the graph is $1.6 T_{C}$.

Figures 18 and 19 are the similar graphs but for $\Upsilon(1 S)$ and $\Upsilon(2 S)$ states, respectively. The dissociation temperature as obtained from the respective Figs. 18 and 19 are $3.2 T_{C}$ and $2.2 T_{C}$ (Table 1).

In Fig. 20, we present the variation of survival probability of $J / \Psi$ and $\Psi^{\prime}$ with respect to $N_{\text {part }}$ at highest RHIC energy,

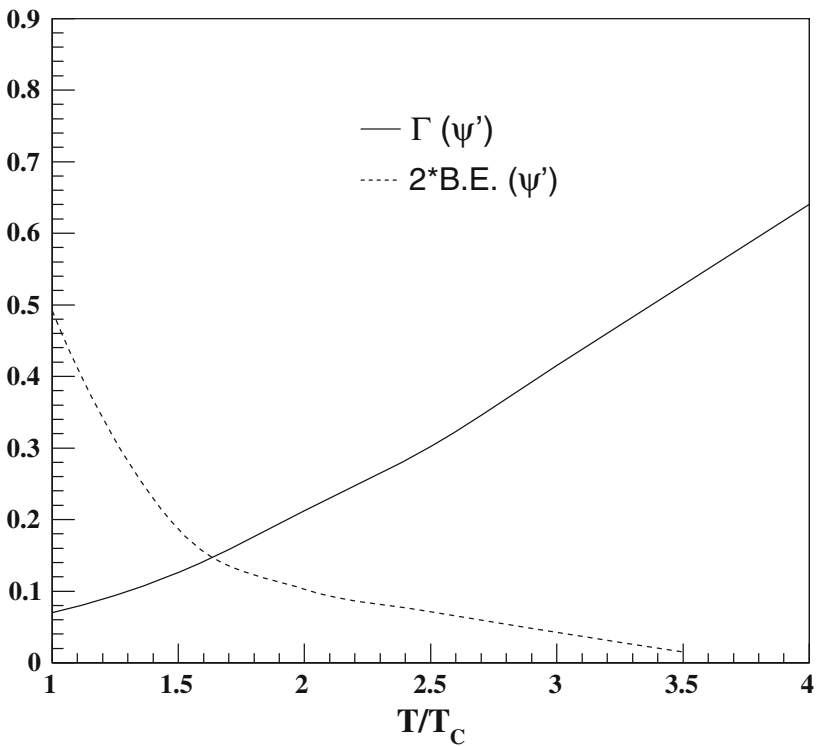

Fig. 17 Variation of two times of binding energy of $\psi^{\prime}$ and decay width with respect to $T / T_{C}$

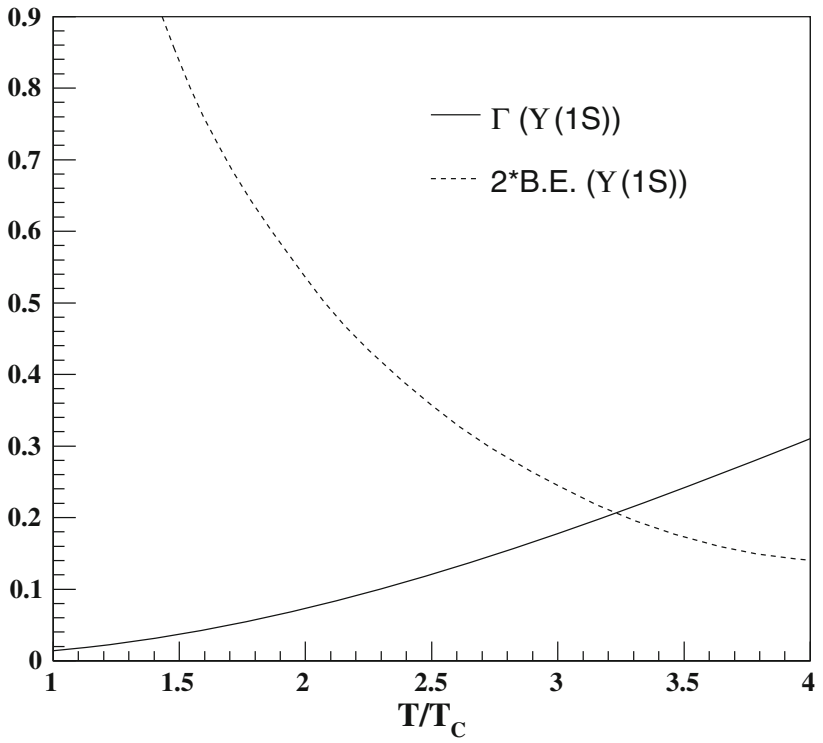

Fig. 18 Variation of two times of binding energy of $\Upsilon(1 S)$ and decay width with respect to $T / T_{C}$

i.e., $\sqrt{s_{N N}}=200 \mathrm{GeV}$. As it is clear from the binding energy and decay width curve of $J / \psi$ and $\psi^{\prime}$, the dissociation probability of $\psi^{\prime}$ is large in comparison to $J / \psi$. Thus the survival probability of $J / \psi$ is larger than the $\psi^{\prime}$ at each centrality. In other words, it means a less suppression of $J / \psi$ in comparison to $\psi^{\prime}$. We have compared our $J / \psi$ results with the corresponding results obtained by STAR experiment [52]. Our model results underestimate the data in most peripheral collisions. However, it suitably describes the data for central and semi-peripheral collisions. Figure 21 shows the variation of survival probability of $J / \psi$ and $\psi^{\prime}$ with respect to cen- 


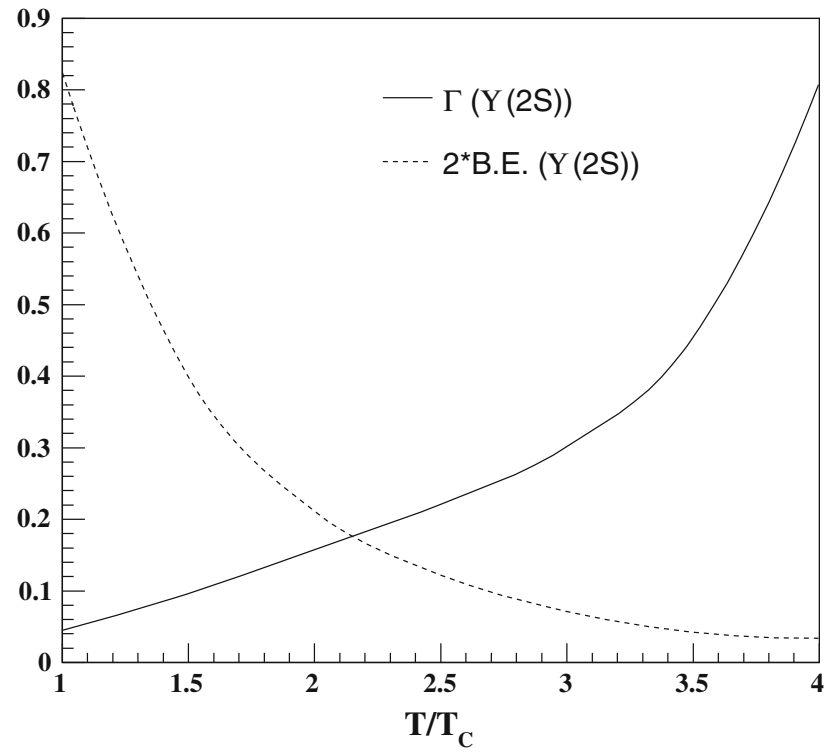

Fig. 19 Variation of two times of binding energy of $\Upsilon(2 S)$ and decay width with respect to $T / T_{C}$

Table 1 Dissociation temperature $\left(T_{d}\right)$ for various quarkonium states

\begin{tabular}{llll}
\hline $\begin{array}{l}\text { Charmonium } \\
\text { states }\end{array}$ & $\begin{array}{l}\text { Diss. temp. } \\
\left(T_{d}\right)\end{array}$ & $\begin{array}{l}\text { Bottomonium } \\
\text { states }\end{array}$ & $\begin{array}{l}\text { Diss. temp. } \\
\left(T_{d}\right)\end{array}$ \\
\hline$J / \psi$ & $2.4 T_{C}$ & $\Upsilon(1 S)$ & $3.2 T_{C}$ \\
$\psi^{\prime}$ & $1.6 T_{C}$ & $\Upsilon(2 S)$ & $2.2 T_{C}$ \\
\hline
\end{tabular}

trality at LHC energy, i.e., $\sqrt{s_{N N}}=2.76 \mathrm{TeV}$. As the energy increases, the corresponding temperature and energy density in each centrality class also increases and thus the survival probability of $J / \psi$ and $\psi^{\prime}$ decreases in comparison to RHIC energy results. We have compared our model result with the experimental data obtained by CMS Collaboration [53,54]. Model results for $J / \psi$ satisfy the data over the entire centrality region except in extreme central collisions. From this plot, one can observe that as we move towards the central collisions from the semiperipheral collisions, there is a small rise in the survival probability. This rise is quite clearly visible in the case of $\psi^{\prime}$. The rise of survival probability in central collisions at LHC energy is due to the rise of regeneration effect through recombination of charm-anticharm pairs in the later stage of the medium evolution. We found in our calculation that the regeneration effect is negligibly small at RHIC energy.

Figures 22 and 23 presents the survival probability of $\Upsilon(1 S)$ and $\Upsilon(2 S)$ at RHIC and LHC energies, respectively. Due to their large mass in comparison to charmonia, the decay width is small and thus the survival probability of $\Upsilon(1 S)$ and $\Upsilon(2 S)$ is large at each $N_{\text {part }}$ in comparison to the survival probability of $J / \psi$ and $\psi^{\prime}$, respectively. We have compared our model results with the experimental data

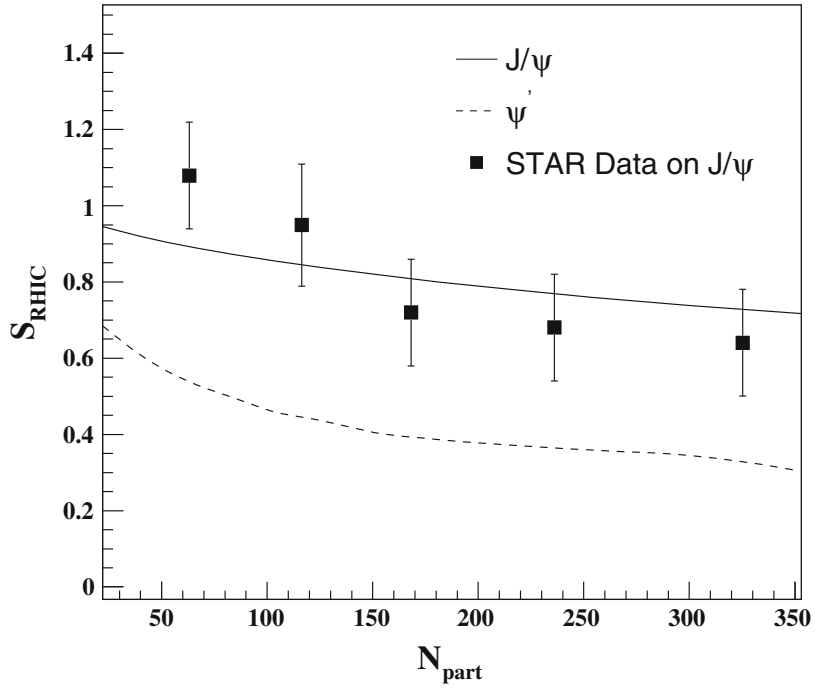

Fig. 20 Variation of survival probability (S) of $J / \psi$ and $\psi^{\prime}$ with respect to $N_{\text {part }}$ at center of mass energy $\sqrt{s_{N N}}=200 \mathrm{GeV}$. Experimental Data is taken from Ref. [52]

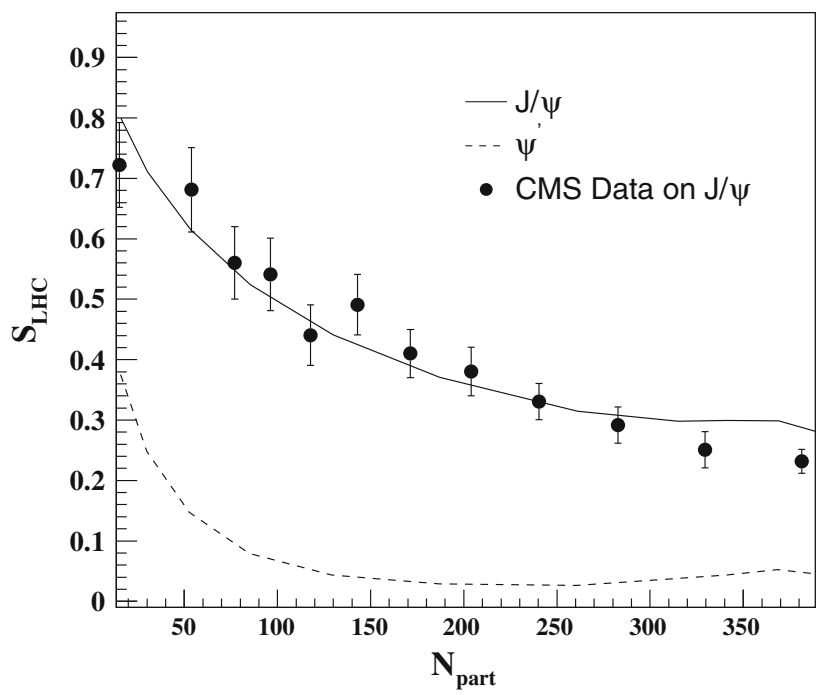

Fig. 21 Variation of survival probability (S) of $J / \psi$ and $\psi^{\prime}$ with respect to $N_{\text {part }}$ at center of mass energy $\sqrt{s_{N N}}=2.76 \mathrm{TeV}$. Experimental Data is taken from Refs. [53,54]

wherever they are available. In Fig. 22, we have shown the STAR data [55,56] for $\Upsilon(1 S)$ and combined suppression of $\Upsilon(1 S)+\Upsilon(2 S)+\Upsilon(3 S)$. Further in Fig. 23, we have plotted the CMS data [53,54] for $\Upsilon(1 S)$ and $\Upsilon(2 S)$. We observed that our model at RHIC energy is able to reproduce the experimental data of $\Upsilon(1 S)$. Further at LHC energy, model results regarding $\Upsilon(1 S)$ and $\Upsilon(2 S)$ satisfy the experimental data satisfactorily. However our results underestimate the data in semi-peripheral collisions. In central collisions, one can again observe the clear effect of regeneration on the survival probability of $\Upsilon(1 S)$ and $\Upsilon(2 S)$ which is negligibly small at RHIC energy. 


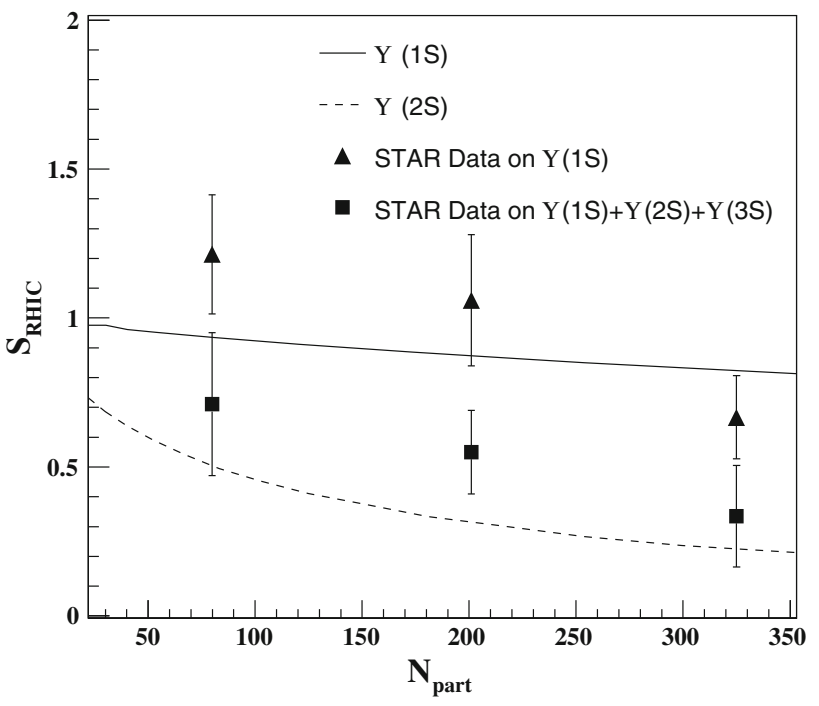

Fig. 22 Variation of survival probability (S) of $\Upsilon(1 S)$ and $\Upsilon(2 S)$ with respect to $N_{\text {part }}$ at center of mass energy $\sqrt{s_{N N}}=200 \mathrm{GeV}$. Experimental Data are taken from Refs. $[55,56]$

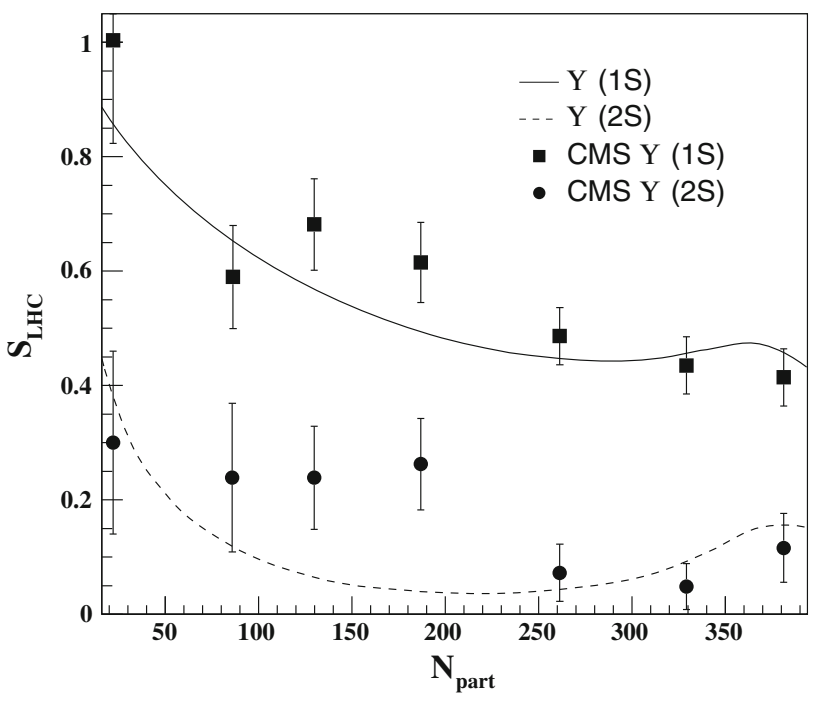

Fig. 23 Variation of survival probability (S) of $\Upsilon(1 S)$ and $\Upsilon(2 S)$ with respect to $N_{\text {part }}$ at center of mass energy $\sqrt{s_{N N}}=2.76 \mathrm{TeV}$. Experimental Data are taken from Refs. [53,54]

Finally we have calculated the double ratio between charmonia states $\left(\psi^{\prime}\right.$ and $\left.J / \psi\right)$ and bottomonia states $(\Upsilon(2 S)$ and $\Upsilon(1 S))$ at $2.76 \mathrm{TeV}$ with respect to $N_{\text {part }}$ in Figs. 24 and 25, respectively. In Fig. 24, we have compared model result for the double ratio of charmonium states with the recent experimental data from CMS Collaboration at mid-rapidity as well as at forward rapidity. Since we have done our calculation for survival probability at mid-rapidity thus one can observe that our model with modified heavy quark potential satisfies the data well in most peripheral collisions while it underestimates the data at semicentral and central collisions. Similarly, in Fig. 25, we have compared our model results

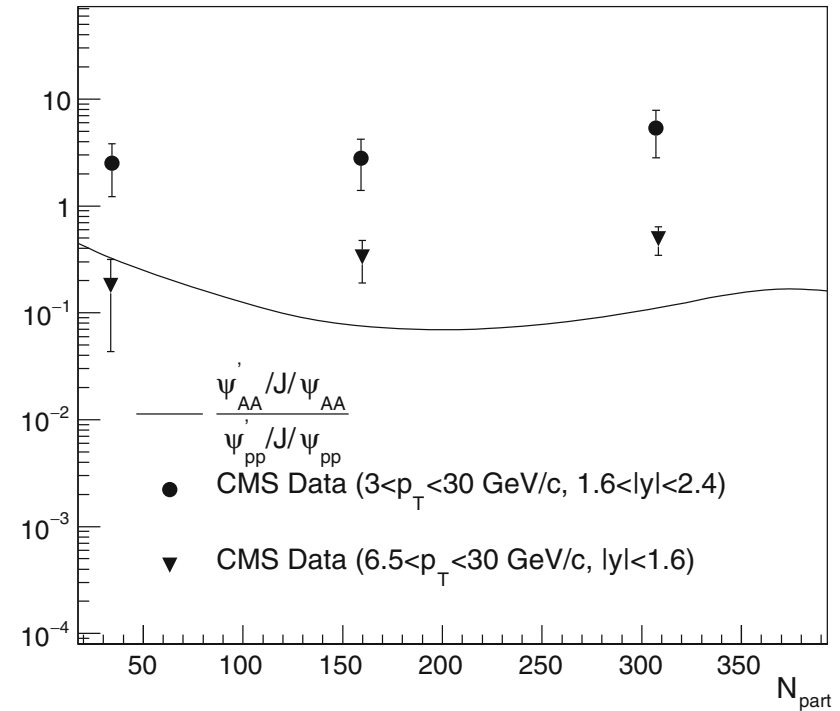

Fig. 24 Variation of survival probability (S) of $\left[\psi^{\prime} / J / \psi\right]_{P b P b} /\left[\psi^{\prime} / J / \psi\right]_{p p}$ with respect to $N_{\text {part }}$ at center of mass energy $\sqrt{s_{N N}}=2.76 \mathrm{TeV}$. Experimental Data are taken from Refs. [53,54]

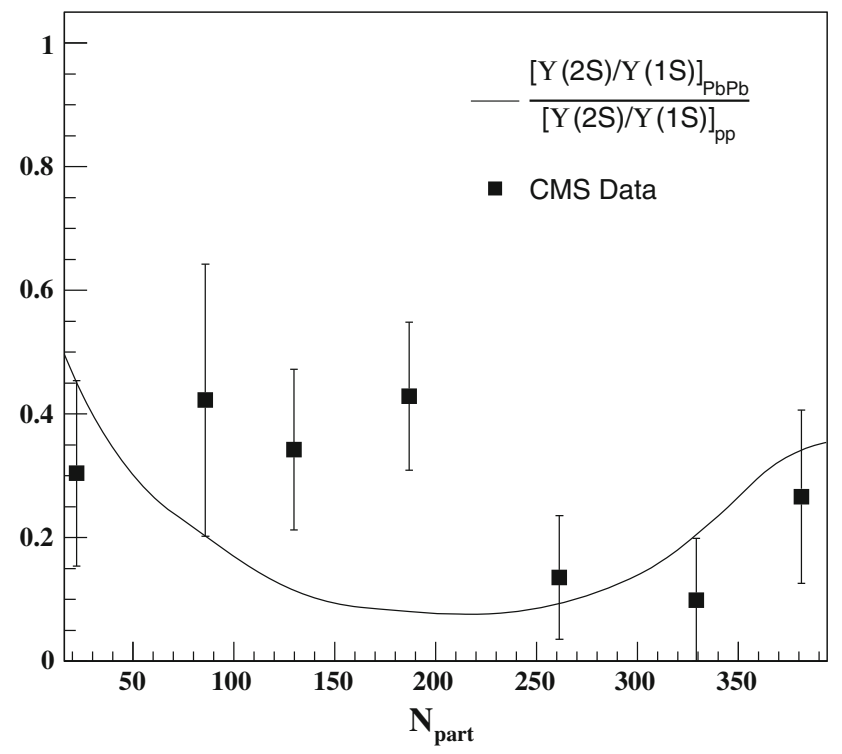

Fig. 25 Variation of survival probability (S) of $[\Upsilon(2 S) / \Upsilon(1 S)]_{P b P b} /[\Upsilon(2 S) / \Upsilon(1 S)]_{p p}$ with respect to $N_{\text {part }}$ at center of mass energy $\sqrt{s_{N N}}=2.76 \mathrm{TeV}$. Experimental Data is taken from Refs. [53,54]

for the double ratio of bottomonium states with the experimental data. We observed that the model results satisfy the data satisfactorily well. Only in the semi-peripheral region, the model underestimates.

In summary, we have solved the $1+1$ dimension Schrodinger equation using a modified heavy quark potential and obtained the eigen function and eigen values of the different charmonium and bottomonium states. We have also calculated the binding energy by solving the $1+1$ 
dimensional Schrodinger equation for an infinite heavy quark potential. Further we have obtained the decay width of different quarkonium states using temperature dependent wavefunction obtained by us and demonstrate their variation with respect to temperature. Furthermore we have obtained the dissociation temperature of different quarkonium states by using the dissociation criteria, i.e., decay width $=2 \times$ binding energy. After that we feed these decay widths in our recently proposed unified model to calculate the survival probability of various quarkonium states. We have also obtained the nuclear modification factor for double ratios and observed that our model suitably describes the experimental data regarding nuclear modification factor (or survival probability).

Acknowledgements PKS acknowledges IIT Ropar, India for providing an institute postdoctoral research grant.

Open Access This article is distributed under the terms of the Creative Commons Attribution 4.0 International License (http://creativecomm ons.org/licenses/by/4.0/), which permits unrestricted use, distribution, and reproduction in any medium, provided you give appropriate credit to the original author(s) and the source, provide a link to the Creative Commons license, and indicate if changes were made. Funded by SCOAP ${ }^{3}$.

\section{References}

1. T. Matsui, H. Satz, Phys. Lett. B 178, 416 (1986)

2. N. Brambilla et al., Eur. Phys. J. C 71, 1534 (2011)

3. F. Karsch, M.G. Mustafa, M.H. Thoma, Phys. Lett. B 497, 249 (2001)

4. A. Mocsy, P. Petreczky, Eur. Phys. J. C 43, 77 (2005)

5. C.Y. Wong, Phys. Rev. C 72, 034906 (2005)

6. D. Cabrera, R. Rapp, Phys. Rev. D 76, 114506 (2007)

7. A. Mocsy, P. Petreczky, Phys. Rev. Lett. 99, 211602 (2007)

8. W.M. Alberico, A. Beraudo, A. De Pace, A. Molinari, Phys. Rev. D 77, 017502 (2008)

9. A. Mocsy, P. Petreczky, Phys. Rev. D 77, 014501 (2008)

10. P. de Forcrand et al., QCD-TARO Collaboration, Phys. Rev. D 63, 054501 (2001)

11. G.T. Bodwin, E. Braaten, G.P. Lepage, Phys. Rev. D 51, 1125 (1995) [Erratum: Phys. Rev. D 55, 5853 (1997)]

12. G.T. Bodwin, Int. J. Mod. Phys. Conf. Ser. 02, 9 (2011)

13. N. Brambilla, A. Pineda, J. Soto, A. Vairo, Nucl. Phys. B 566, 275 (2000)

14. N. Brambilla, A. Pineda, J. Soto, A. Vairo, Rev. Mod. Phys. 77, 1423 (2005)

15. N. Brambilla, J. Ghiglieri, A. Vairo, P. Petreczky, Phys. Rev. D 78, 014017 (2008)

16. M. Laine, O. Philipsen, P. Romatschke, M. Tassler, JHEP 0703, 054 (2007)

17. L. Thakur, U. Kakade, B.K. Patra, Phys. Rev. D 89, 094020 (2014)
18. M. Margotta, K. McCarty, C. McGahan, M. Strickland, D. YagerElorriaga, Phys. Rev. D 83, 105019 (2011)

19. M. Strickland, D. Bazow, Nucl. Phys. A 879, 25 (2012)

20. F. Karsch, J. Phys. Conf. Ser. 46, 122 (2006). arXiv:hep-lat/0608003

21. M. Cheng et al., Phys. Rev. D 78, 034506 (2008)

22. Y. Maezawa et al., WHOT-QCD Collaboration, Phys. Rev. D 75, 074501 (2007)

23. O. Andreev, V.I. Zakharov, Phys. Lett. B 645, 437 (2007)

24. V. Agotiya, V. Chandra, B.K. Patra, Phys. Rev. C 80, 025210 (2009)

25. L. Thakur, N. Haque, U. Kakade, B.K. Patra, Phys. Rev. D 88, $054022(2013)$

26. L. Thakur, N. Haque, H. Mishra, Phys. Rev. D 95(3), 036014 (2017)

27. Y. Burnier, A. Rothkopf, Phys. Lett. B 753, 232 (2016)

28. V.K. Agotiya, V. Chandra, M.Y. Jamal, I. Nilima, Phys. Rev. D 94(9), 094006 (2016)

29. S. Ganesh, M. Mishra, Phys. Rev. C 91(3), 034901 (2015)

30. C.R. Singh, P.K. Srivastava, S. Ganesh, M. Mishra, Phys. Rev. C 92, 034916 (2015)

31. B.K. Patra, V. Agotiya, V. Chandra, Eur. Phys. J. C 67, 465 (2010)

32. U. Kakade, B.K. Patra, L. Thakur, Int. J. Mod. Phys. A 30(09), $1550043(2015)$

33. R.L. Thews, Eur. Phys. J. C 43, 97 (2005)

34. R.L. Thews, Nucl. Phys. A 702, 341 (2002)

35. R.L. Thews, J. Phys. G 30, S369 (2004)

36. B. Krouppa, A. Rothkopf, M. Strickland, Phys. Rev. D 97, 016017 (2018)

37. J.P. Blaizot, D. DeBoni, P. Faccioli, G. Garberoglio, Nucl. Phys. A 946, 49 (2016)

38. A. Rothkopf, T. Hatsuda, S. Sasaki, Phys. Rev. Lett. 108, 162001 (2012)

39. Y. Burnier, A. Rothkopf, Phys. Rev. D 95, 054511 (2017)

40. Y. Burnier, A. Rothkopf, Phys. Rev. D 86, 051503(R) (2012)

41. M. Ali-Akbari, D. Giataganas, Z. Rezaei, Phys. Rev. D 90, 086001 (2014)

42. B.K. Patra, H. Khanchandani, L. Thakur, Phys. Rev. D 92, 085034 (2015)

43. H. Liu, K. Rajagopal, U.A. Wiedemann, J. High Energy Phys. 03, 066 (2007)

44. K.B. Fadafan, D. Giataganas, H. Soltanpanahi, J. High Energy Phys. 11, 107 (2013)

45. S. Tahery, J. Sadeghi, J. Phys. G 44, 105001 (2017)

46. S. Digal, O. Kaczmarek, F. Karsch, H. Satz, Eur. Phys. J. C 43, 71 (2005)

47. H. Satz, J. Phys. G 32, R25 (2006)

48. P.K. Srivastava, S.K. Tiwari, C.P. Singh, Phys. Rev. C 88, 044902 (2013)

49. P.K. Srivastava, M. Mishra, C.P. Singh, Phys. Rev. C 87, 034903 (2013)

50. S. Ganesh, M. Mishra, Phys. Rev. C 88, 044908 (2013)

51. The ATLAS Collaboration, CERN-PH-EP-2014-172

52. L. Adamczyk et al., STAR Collaboration, Phys. Lett. B 722, 55 (2013)

53. M. Murray, CMS Collaboration, Nucl. Phys. Proc. Suppl. 233, 212 (2012)

54. H. Kim, CMS Collaboration, J. Phys. Conf. Ser. 422, 012015 (2013)

55. L. Adamczyk et al., STAR Collaboration, Phys. Lett. B 735, 127 (2014)

56. L. Adamczyk et al., STAR Collaboration, Phys. Lett. B 743, 537 (2015) 